\title{
Is the Fano Antiresonance a Necessary Requirement for Circulating Currents in Mesoscopic Interferometers?
}

\author{
Yao Heng Su, Sam Young Cho, $*$ and Ai Min Chen \\ Centre for Modern Physics and Department of Physics, \\ Chongqing University, Chongqing 400030, China \\ Taeseung Choi \\ Barom Liberal Arts College, Seoul Women's University, Seoul, 139-774, Korea
}

(Dated: November 8, 2018)

\begin{abstract}
Coherent quantum tunneling effects on quantum interference are investigated in electron transport through a mesoscopic interferometer. An evanescent wave tunneling through a potential barrier in one arm can interfere with a propagating wave passing through the other arm of interferometer. It is shown that, even for the same arm lengths, such a quantum interference can induce a circulating current, where Fano antiresonances do not occur in electron transmission. It is found that there exists a critical value of asymmetric arm lengths that gives rise to a Fano antiresonance in electron transmission for the quantum interference between evanescent and propagating waves. We discuss the effects of Fano antiresonances originating from the asymmetric arm lengths on circulating currents.
\end{abstract}

PACS numbers: 73.63.-b, 85.35.-p, 85.35.Ds

Keywords: Quantum interferometer, Quantum transport, Circulating current, Fano antiresonance, Evanescent wave

*Electronic address: sycho@cqu.edu.cn; Fax: +86-23-65111531 


\section{INTRODUCTION}

Nonadditivity [1 -3] of parallel conductances is a prototype of quantum coherence in mesosocpic electronics [4]. It also implies that some classical circuit laws may not be valid due to nonlocality of electron waves in mesoscopic interferometers. As an another example, a recent theoretical study has shown that a circulating current [5] via a so-called current magnification and negative current can flow along the loop path of interferometers owing to quantum interference even in the absence of magnetic fields, which cannot occur in classical parallel resistors. The quantum interference for the circulating current has been interpreted as a Fano-type interference [6] with its characteristic transmission zeros [7], so called Fano antiresonance, in electron transport through interferometers only with an asymmetric arm lengths.

The unique behaviors of circulating currents have been studied in various types of electronic interferometers such as single loop interferometers with a stub [8] or impurity potential [9, 10] embedded in one of arms, an evanescent wave interferometer with a potential well [11], a multichannel interferometer with an impurity [12], double loop interferometers [13, 14], double quantum dot interferometers [15, 16], multiple-arm interferometers [17, 18], and spindependent interferometers [18-20]. In such interferometers, a circulating heat [16] and spin [18 20] currents have been reported as well as electric circulating currents. However, almost all such studies have shown that the Fano antiresonances mediate such a circulating current in only geometrically asymmetric mesoscopic interferometers. Thus, it may be believed that the Fano antiresonances in electron transmission through mesoscopic interferometers are a necessary requirement for the existence of circulating currents. However, in an asymmetric

multichannel interferometer with a potential impurity [12], it was found that a circulating current can be induced without Fano antiresonances in a special energy range where there are two propagating modes with a bound state.

It is not clear, to our knowledge, whether a Fano antiresonance is a necessary requirement for circulating currents in such two-terminal mesoscopic interferometers. To help our understanding on the question and to give a better understanding on fundamental quantum interference, in this study, we consider a realizable simplest model (see Fig. 1), that is a single loop interferometer with a potential barrier in each arms, which is enough to capture an essential physics. We focus on the quantum interference between propagating and 
evanescent waves respectively flowing through each arms of the interferometer. It is found that a circulating current can be induced by the quantum interference without transmission antiresonances even for the symmetric arm length of interferometer. For the circulating currents, the tunneling current through the potential barrier flows against the applied bias while a propagating current magnification occurs in the other arm. These behaviors of circulating currents persist up to a critical value of asymmetric arm lengths where electron transmission starts to have a Fano antiresonance. The effects of Fano antiresonances due to the asymmetric arm lengths are discussed on circulating currents.

\section{MODEL}

We start with an ideal loop interferometer with a single channel in the absence of magnetic fields in the top panel of Fig. 1. The interferometer is connected to two reservoirs where electron can be injected to the interferometer through the ideal leads $L$ and $R$ when the chemical potentials are not equal each other, $\mu_{R} \neq \mu_{L}$. The geometrical symmetry of interferometer is determined by its upper and lower arm lengths $l_{1}$ and $l_{2}$. If the loop has a narrower transverse width than the ideal leads, an injected electron momenta cannot be matched with the electron energy in the loop arms due to their narrower transverse confinement. Thus, in the arm, electron undergoes a decaying of wavefunction, i.e., an evanescent wave. This phenomena can be well described by introducing arm potentials $V_{1}$ and $V_{2}$ respectively for the upper and lower arms within one-dimensional waveguide theory [21]. These potentials can make the energy levels of the upper and lower arms higher than those of the ideal leads. For $V_{2}<V_{1}$, the energy level diagrams are depicted in the bottom panel of Fig. 1.

We concentrate on the energy region (ii) $V_{2}<E_{F}<V_{1}$ in the bottom panel of Fig. 1. where the quantum interference between propagating and evanescent waves occurs. We employ one-dimensional waveguide theory [21] with local coordinates. The Hamiltonians are given as $H_{L, R}=p^{2} / 2 m^{*}$ for the left and right leads and $H_{1,2}=p^{2} / 2 m^{*}+V_{1,2}$ for the upper and lower arms, where $p$ is the electron momentum, $m^{*}$ is the electron effective mass, and $V_{1,2}$ are the electric potentials in the upper and lower arms, respectively. Suppose that an electron is injected from the left lead with its energy $E_{F}$. The wavefunctions for each regions are respectively written as $\Psi_{L}(x)=e^{i k_{F} x}+r e^{-i k_{F} x}$ for the left lead, $\Psi_{1}(x)=a e^{-\kappa_{F} x}+b e^{\kappa_{F} x}$ for 

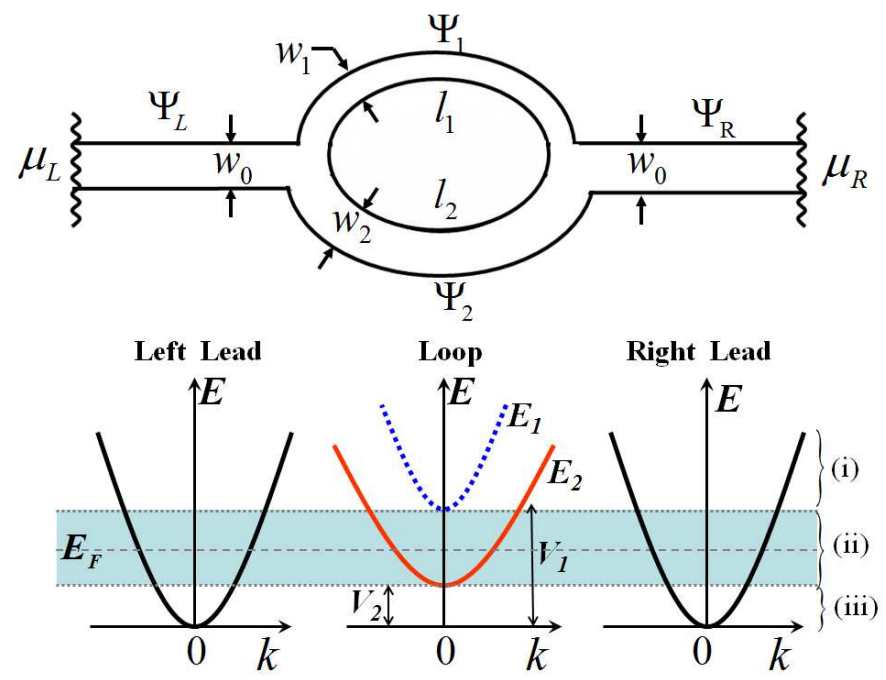

FIG. 1: (color online) Top: Mesosocopic interferometers coupled to the left and right electron reservoirs that are respectively characterized by chemical potentials $\mu_{L}$ and $\mu_{R} . l_{1}$ and $l_{2}$ denote the upper and lower arm length, respectively, such that the loop size of interferometer is $L=l_{1}+l_{2}$. $\Psi$ 's are the electron wavefunctions in each regions. Bottom: Energy level diagram for each regions. If the loop has a narrower transverse width than the ideal leads, the energy bands $E_{1}$ and $E_{2}$ in the upper and lower arms can be shifted up to have a higher energy than the energy bands in the leads, which can be modeled by introducing potential barriers $V_{i}(i \in\{1,2\})$ in each arms within one-dimensional waveguide theory. Here, the energies in the arms are depicted for $V_{2}<V_{1}$. Quantum interferences in this interferometer can be characterized by three energy regimes. (i) For $0<E_{F}<V_{i}$, electron can tunnel through each arms as an evanescent wave. (ii) For $V_{i}<E_{F}<V_{j}$ $(i \neq j)$ in the shaded energy regime, electron propagates in one arm and tunnels through the other arm as an evanescent wave. (iii) For $V_{j}<E_{F}$, electron propagates through the interferometer with different momentum $\left(V_{1} \neq V_{2}\right)$. This study is focused on the case (ii).

the upper arm, $\Psi_{2}(x)=c e^{i k_{F}^{\prime} x}+d e^{-i k_{F}^{\prime} x}$ for the lower arm, and $\Psi_{R}(x)=t e^{i k_{F} x}$ for the right lead, where $k_{F}=\sqrt{2 m^{*} E_{F}} / \hbar, \kappa_{F}=\sqrt{K_{1}^{2}-k_{F}^{2}}$, and $k_{F}^{\prime}=\sqrt{k_{F}^{2}-K_{2}^{2}}$ with $K_{i}=\sqrt{2 m^{*} V_{i}} / \hbar$ $(i \in\{1,2\})$. By using the Griffith boundary conditions [22] (current conservation) and the continuities of wavefunctions at the left and right junctions, one can obtain the coefficients of wavefunctions $r, a, b, c, d$, and $t$ in terms of $k_{F}, \kappa_{F}, k_{F}^{\prime}, l_{1}$, and $l_{2}$.

The probability current densities $J_{j}(x)=\frac{e \hbar}{2 m i}\left(\Psi_{j}^{*}(x) \partial_{x} \Psi_{j}(x)-\Psi_{j}(x) \partial_{x} \Psi_{j}^{*}(x)\right)(j \in$ $\{L, 1,2, R\})$ through the whole interferometer and the upper and lower arms can be re- 
spectively obtained as

$$
\begin{aligned}
J & =J_{0} \mathcal{F}_{0}\left(\mathcal{F}_{1}-\mathcal{F}_{2}\right)^{2}, \\
J_{1} & =J_{0} \mathcal{F}_{0}\left(\mathcal{F}_{1}-\mathcal{F}_{2}\right) \mathcal{F}_{1}, \\
J_{2} & =J_{0} \mathcal{F}_{0}\left(\mathcal{F}_{2}-\mathcal{F}_{1}\right) \mathcal{F}_{2},
\end{aligned}
$$

where $J_{0}=e \hbar k_{F} / m^{*}$ is the unit probability current density. Here, $\mathcal{F}_{0}=4 k_{F}^{2} \kappa_{F}^{2} k_{F}^{\prime 2} /\left[4 k_{F}^{2}\left(\kappa_{F} \cosh \kappa_{F} l_{1} \sin k_{F}^{\prime} l_{2}+k_{F}^{\prime} \sinh \kappa_{F} l_{1} \cos k_{F}^{\prime} l_{2}\right)^{2}+\left(\left(k_{F}^{2}-\kappa_{F}^{2}+\right.\right.\right.$ $\left.\left.\left.k_{F}^{\prime 2}\right) \sinh \kappa_{F} l_{1} \sin k_{F}^{\prime} l_{2}+2 \kappa_{F} k_{F}^{\prime}\left(1-\cosh \kappa_{F} l_{1} \cos k_{F}^{\prime} l_{2}\right)\right)^{2}\right], \quad k_{F}^{\prime} \mathcal{F}_{1}=\sin k_{F}^{\prime} l_{2}, \quad$ and $\kappa_{F} \mathcal{F}_{2}=-\sinh \kappa_{F} l_{1}$. The transmission amplitude is also written as $T=J / J_{0}=\mathcal{F}_{0}\left(\mathcal{F}_{1}-\mathcal{F}_{2}\right)^{2}$.

\section{CIRCULATING CURRENT AND ITS GENERATING CONDITION}

A transport current $J$ flows from the left to the right, as assumed $\mu_{R}<\mu_{L}$, and is split into two local currents $J_{1}$ and $J_{2}$ respectively flowing in the upper and lower arms under the current conservation. If $J_{1}\left(J_{2}\right)$ is bigger than $J, J_{2}\left(J_{1}\right)$ should flow against applied bias due to the current conservation [5]. These phenomenon can be understood by introducing a circulating current $J_{c}$ flowing along the loop path of interferometer. Consequently, the arguments of circulating current can be summarized by the expression [13] of circulating current

$$
J_{c}=\frac{\operatorname{sign}\left[J_{1}\right]}{2}\left(\left|J_{1}\right|+\left|J_{2}\right|-J\right)
$$

Equation (2) shows that any classical parallel resistor cannot have a circulating current. The definition then allows to capture a pure quantum mechanical effect for electron transport through two-terminal interferometer.

According to the arguments of circulating current with the current expressions in Eqs. (1a)-(1c), one can obtain a generating condition of circulating current as a function of parameters $\left\{K_{1} L, K_{2} L, \beta, k_{F} L\right\}$, where $\beta=l_{1} / l_{2}$ characterizes the geometrical asymmetry of interferometer. $\beta=1$ denotes a geometrically symmetric interferometer. If $J<J_{1}$ and $J_{2}<0$, one can assign a circulating current by the generating condition written as

$$
\mathcal{F}_{1}\left(K_{2} L, \beta, k_{F} L\right)<\mathcal{F}_{2}\left(K_{1} L, \beta, k_{F} L\right)<0
$$


From Eq. (2), circulating currents satisfying Eq. (3a) flow in clockwise direction. If $J<J_{2}$ and $J_{1}<0$, the generating condition for counterclockwise circulating current is given as

$$
\mathcal{F}_{2}\left(K_{1} L, \beta, k_{F} L\right)<\mathcal{F}_{1}\left(K_{2} L, \beta, k_{F} L\right)<0 .
$$

\section{CIRCULATING CURRENT WITHOUT FANO ANTIRESONANCES FOR SYMMETRIC ARM LENGTH}

Let us consider first the symmetric arm length $l_{1}=l_{2}$ for $K_{1} L \neq 0$ and $K_{2} L=0$. We plot the transmission amplitudes $T=J / J_{0}$ and circulating currents $J_{c} / J_{0}$ as a function of $k_{F} L$ in Figs. 2 (a) and (b), respectively. Note that no transmission zeros are seen in Fig. 2 (a). However, Fig. 2 (b) shows that there exist circulating currents even in the geometrically symmetric interferometers. Also, our circulating current shows its very different behaviors from the ones of geometrically asymmetric interferometers, showing that Fano antiresonances in electron transmission accompany a circulating current, in Refs. [5, 8 -11, 17 20].

The characteristic properties of our circulating current in geometrically symmetric interferometer for the quantum interference between propagating and evanescent waves are summarized as follows: (i) From $\mathcal{F}_{2} \supsetneqq \mathcal{F}_{1}$ for $\beta=1$ and $\mathcal{F}_{2}<\mathcal{F}_{1}<0$ in Eq. (3b) , the generating condition of circulating current becomes $\mathcal{F}_{1}<0$. Thus, a circulating current exists in the regions $(2(2 m-1) \pi, 4 m \pi)$ of $k_{F} L$ for $4 m \pi \leq K_{1} L \leq 2(2 m+1) \pi$ or in the regions $\left(2(2 m-1) \pi, K_{1} L\right)$ of $k_{F} L$ for $2(2 m-1) \pi \leq K_{1} L \leq 4 m \pi$. Here, $m$, a positive integer, corresponds to the $m$-th transmission resonance for the range $k_{F} L((2 m-1) \pi, 2 m \pi)$. Hence, the $2 m$-th transmission resonance accompany a circulating current. This interesting behavior can be understood by a following way. Suppose that there is an isolated loop with a high potential which forms a quantum well in the loop. For our interferometer, the eigen energies of the quantum well in the isolated loop may roughly one-to-one correspond to the resonant transmission energies $E_{m} \sim \alpha \frac{\hbar^{2}}{2 m^{*} L^{2}}(2 m \pi)^{2}$, where a parameter $\alpha$ is responsible for the effect of coupling to the leads and must be smaller than $1(0<\alpha<1)$. If the higher

potential $V_{1}$ (here, $K_{1} L$ ) becomes bigger, the resonant energies become closer to the eigen energies of the quantum well, which is shown in Fig. 2 (a). Also, in the coordinate of isolated loop, the $2 m$-th eigen state of the quantum well has a odd parity wavefucntion while the $(2 m-1)$-th eigen state has a even parity wavefunction. Then, our circulating currents may be mediated by the odd parity (anti-symmetric) wavefunctions of the quantum well. (ii) A 

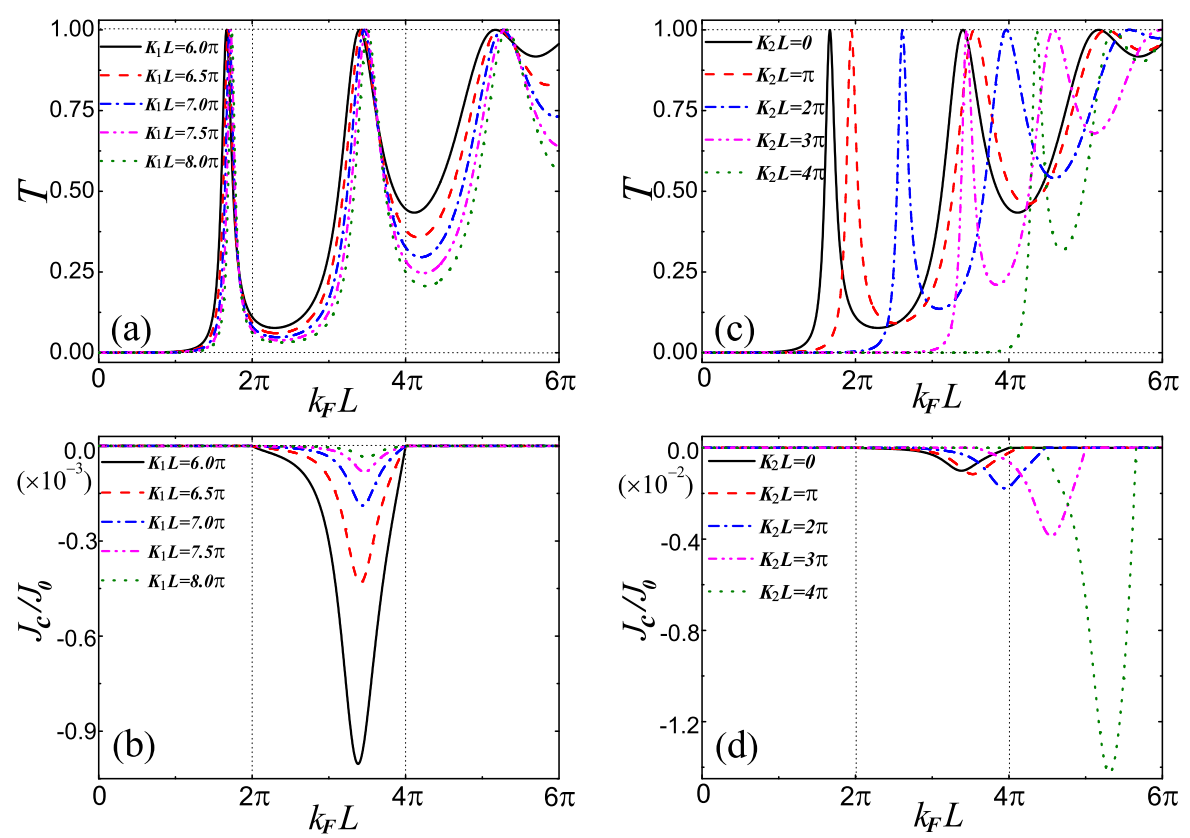

FIG. 2: (color online) Transmission amplitudes $T$ and circulating currents $J_{c} / J_{0}$ as a function of $k_{F} L$ for the symmetric arm length $l_{1}=l_{2}$. Here, $J_{0}=e \hbar k_{F} / m^{*}$ and $K_{i}=\sqrt{2 m^{*} V_{i}} / \hbar(i \in\{1,2\})$. Note that no transmission zeros are seen in (a) and (c). Left: (a) $T=J / J_{0}$ and (b) $J_{c} / J_{0}$ for various values of $K_{1} L$ and $K_{2} L=0$. As $K_{1} L$ increases from $K_{1} L=6 \pi$, a smaller circulating current flows in the same range $(2 \pi, 4 \pi)$ of $k_{F} L$. Right: (c) $T=J / J_{0}$ and (d) $J_{c} / J_{0}$ for $K_{1} L=6 \pi$ and various values of $K_{2} L$. As $K_{2} L$ increases with $K_{1} L=6 \pi$, a larger circulating current is seen in the shifted ranges $\left(\sqrt{(2 \pi)^{2}+\left(K_{2} L\right)^{2}}, \sqrt{(4 \pi)^{2}+\left(K_{2} L\right)^{2}}\right)$ of $k L$ for the chosen parameters. If one choose a value of $K_{2} L$ in the range $\sqrt{32} \pi<K_{2} L<K_{1} L=6 \pi$, no circulating current can exist.

tunneling current $J_{1}$ flows only against the applied bias in the regions. Compared to the circulating current in the previous studies of geometrically asymmetric interferometers, our circulating current can flow only in one direction, i.e., electron circulates in counterclockwise (clockwise) direction for $V_{1}>V_{2}\left(V_{1}<V_{2}\right)$ in the geometrically symmetric interferometer $\left(l_{1}=l_{2}\right)$. In other words, the asymmetric potential barriers in the interferometer determines the direction of circulating current. (iii) A bigger propagating current $J_{2}$ than transport current $J$ flows in the other arm. (iv) The amplitude of circulating currents decreases as $K_{1} L$ increases in a given range of $k_{F} L$ where a circulating current can exist.

For $K_{2} L \neq 0$ and $K_{1} L=6 \pi$, we display the transmission amplitudes $T=J / J_{0}$ and circulating currents $J_{c} / J_{0}$ as a function of $k L$ in Fig. 2 (c) and (d), respectively. Figure 
22 (d) shows that no circulating current is induced when two evanescent waves interfere for $0<E_{F}<V_{2}<V_{1}$. When propagating and evanescent waves interfere for $V_{2}<E_{F}<V_{1}$, as $V_{2}$ increases up to $K_{2} L=\sqrt{32} \pi$ from zero, the region of circulating current changes to a shifted and narrower region $\left(\sqrt{(2 \pi)^{2}+\left(K_{2} L\right)^{2}}, \sqrt{(4 \pi)^{2}+\left(K_{2} L\right)^{2}}\right)$ from $(2 \pi, 4 \pi)$ in $k_{F} L$ and a larger circulating current can flow. However, it should be noted that as $V_{2}$ increases further to the value of $\sqrt{32} \pi<K_{2} L<K_{1} L=6 \pi$, circulating current cannot be induced.

\section{A CRITICAL VALUE OF ASYMMETRIC ARM LENGTHS AND FANO AN- TIRESONANCES}

Now, let us discuss about Fano antiresonances and their effects on circulating currents in the geometrically asymmetric interferometer $\left(l_{1} \neq l_{2}\right)$. From Eq. (1a), one finds the antiresonance condition given as

$$
\mathcal{F}_{1}\left(K_{2} L, \beta, k_{F} L\right)=\mathcal{F}_{2}\left(K_{1} L, \beta, k_{F} L\right)
$$

At the antiresonances, from Eqs. (1a)-(1c), and (4), the circulating currents also vanish as well as the transport currents. For a given $K_{1} L$ and $K_{2} L$, there can be $s$ solution sets $\left\{k_{F}^{(s)} L\right\}$ satisfying Eq. (4) at a certain value $\beta^{(s)}$, where $s$ is a positive integer, which implies that $s$ Fano antiresonances occur in electron transmission.

For instance, in Fig. 3, we consider the case of $K_{1} L=6 \pi$ and $K_{2} L=0$ and plot $T, J_{c} / J_{0}$, $\mathcal{F}_{1}$, and $\mathcal{F}_{2}$ as a function of $k_{F} L$ for various asymmetric arm lengths (a) $\beta=1 / 7$, (b) $\beta=$ $\beta_{c} \simeq 0.121704$, and (c) $\beta=1 / 9$. In $k_{F} L$, there are the periodic existing ranges of circulating currents from the generating condition Eqs. (3a) and (3b) since $\mathcal{F}_{1}$ is a sinusoidal function of $k_{F} L$ while $\mathcal{F}_{2}$ is a negative monotonic function of $k_{F} L$. (i) For $\beta>\beta^{(1)}=\beta_{c}$, no Fano antiresonance occurs because $\mathcal{F}_{2}<\mathcal{F}_{1}$ in $k_{F} L$ (Fig. 3 (a)). The existing ranges of circulating currents are determined by $\mathcal{F}_{1}(\beta)<0$. The circulating currents flows in the counterclockwise direction. (ii) For $\beta=\beta_{c}$, eventually, a Fano antiresonance appears at $k_{F}^{c} L$. Note that $k_{F}^{c} L$ $\left(C\right.$ in Fig. 3 (b)) locates inside between the first existing range $k_{F} L\left(A^{\prime}, B^{\prime}\right)$ determined by $\mathcal{F}_{1}\left(\beta_{c}\right)<0$. Thus, when $k_{F} L$ is swept across the antiresonance, the direction of circulating current does not changed and the circulating current just disappear at $k_{F}^{c} L$. (iii) As $\beta$ decreases further for $\beta^{(3)}<\beta<\beta^{(1)}$, the electron transmission has two Fano antiresonances inside between the first existing range determined by $\mathcal{F}_{1}\left(\beta^{(3)}<\beta<\beta^{(1)}\right)<0$. Figure 3 (c) 

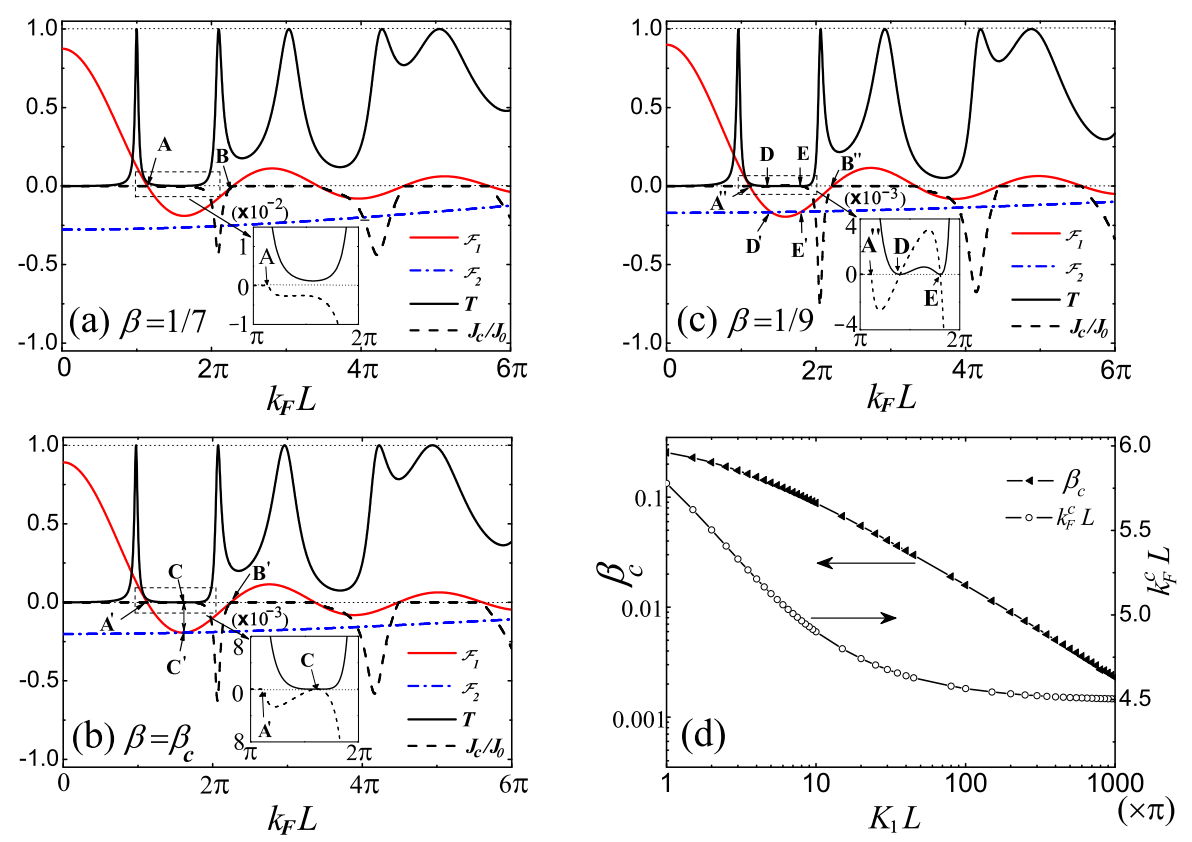

FIG. 3: (color online) Transmission amplitudes $T$ (black solid lines), circulating currents $J_{c} / J_{0}$ (black dashed lines), and the characteristic functions $\mathcal{F}_{1}$ (red solid lines) and $\mathcal{F}_{2}$ (blue dash-dotted lines) as a function of $k_{F} L$ for (a) $\beta\left(=l_{1} / l_{2}\right)=1 / 7$ (no Fano antiresonance), (b) $\beta=\beta_{c} \simeq 0.121704$ (one Fano antiresonance), and (c) $\beta=1 / 9$ (two Fano antiresonances) in the interferometer with an asymmetric arm length $l_{1} \neq l_{2}$. Here, $K_{1} L=6 \pi$ and $K_{2} L=0$. For $\mathcal{F}_{1}=0, J_{c}=0$, e.g., here at (a) $A$ and $B$ of $k_{F} L$, (b) $A^{\prime}$ and $B^{\prime}$ of $k_{F} L$, and (c) $A^{\prime \prime}$ and $B^{\prime \prime}$ of $k_{F} L$. At these $k_{F} L, T \neq 0$. For $\mathcal{F}_{2}<\mathcal{F}_{1}<0, J_{c}<0$ and, for $\mathcal{F}_{1}<\mathcal{F}_{2}<0, J_{c}>0$. For $\mathcal{F}_{1}=\mathcal{F}_{2}$, the Fano antiresonances $(T=0)$ occur and $J_{c}=0$ at the points $C$ in (b) and $D$ and $E$ in (c), which are shown from the pictorial representations of solutions. (d) Critical values $\beta=\beta_{c}$ and $k_{F} L=k_{F}^{c} L$ as a function of $K_{1} L$ with $K_{2} L=0$, where only one Fano antiresonance occurs in electron transmission.

shows that the circulating current direction is changed to the opposite (clockwise) direction in $k_{F} L(D, E)$ between the pair of antiresonances. As the ordered pairs of antiresonances is split to approach closer to the transmission resonances by decreasing further smaller $\beta$, a larger circulating current flows.

For $\beta^{(2 s+1)}<\beta<\beta^{(2 s-1)}, 2 s$ Fano antiresonances occur and the circulating current direction in $k_{F} L$ between the ordered pairs of antiresonances is changed to the opposite direction. Note that the pairs of antiresonances exist inside between the existing ranges of circulating currents determined by $\mathcal{F}_{1}(\beta)<0$. Therefore, the $2 m$-th transmission resonances 
play a significant role for a circulating current even though Fano antiresonances occur in between the ordered pairs of antiresonances of electron transmission where the circulating current direction is changed to the clockwise direction from the counterclockwise direction.

Figure 3 (d) shows that Fano antiresonances can occur in electron transmission through the interferometer when the arm length of propagating wave is longer than that of evanescent wave, i.e., $l_{1}<l_{2}$, As $K_{1} L$ increases, $\beta_{c}$ decreases exponentially and $k_{F}^{c} L$ approaches to about 4.5 .

\section{CONCLUSIONS}

Coherent quantum tunneling effects on quantum interference in a mesoscopic interferometer have been investigated to address whether a circulating current does require a Fano antiresonance in electron transmission. It is found that the quantum interference between the propagating and evanescent waves can induce a circulating current without transmission zeros even for the symmetric arm length of interferometer. A Fano antiresonance of electron transmission was shown to start appearing at a critical value of asymmetric arm lengths. As a result, it was shown that Fano antiresonance is not a necessary requirement for circulating currents in two-terminal mesoscopic interferometers.

\section{Acknowledgments}

We thank Huan-Qiang Zhou for helpful discussions. This work was supported by the NSFC under Grant No.10874252 and Natural Science Foundation Project of CQ CSTC. T. Choi was supported by a research grant from Seoul Women's University(2009).

[1] R. A. Webb, S. Washburn, C. P. Umbach and R. B. Laibowitz, Phys. Rev. Lett. 54, 2696 (1985).

[2] S. Washburn and R. A. Webb, Rep. Prog. Phys. 55, 1311 (1992).

[3] A. Fuhrer, S. Lüscher, T. Ihn, T.Heinzel, K. Ensslin, W. Wegscheider and M. Bichler, Nature 413, $822(2001)$. 
[4] See, for a review,Y. Imry, Introduction to Mesoscopic Physics, 2nd ed. (Oxford University Press, Oxford, 2002); S. Datta, Electronic Transport in Mesoscopic Systems (Cambridge University Press, New York, 1995).

[5] A. M. Jayannavar and P. S. Deo, Phys. Rev. B 51, 10175 (1995).

[6] U. Fano, Phys. Rev. 124, 1866 (1961).

[7] E. Tekman and P. F. Bagwell, Phys. Rev. B 48, 2553 (1993).

[8] S. Y. Cho, T. Choi and C.-M. Ryu, Int. J. Mod. Phys. B 10, 3569 (1996).

[9] T. P. Pareek, P. S. Deo and A. M. Jayannavar, Phys. Rev. B 52, 14657 (1995)

[10] V. Vargiamidis and H. M. Polatoglou, Phys. Rev. B 74, 235323 (2006).

[11] C. Benjamin and A. M. Jayannavar, Phys. Rev. B 68, 085325 (2003).

[12] S. Bandopadhyay, P. S. Deo and A. M. Jayannavar, Phys. Rev. B 70, 075315 (2004).

[13] There is a missing factor, $\operatorname{sign}\left[J_{1}\right]$ or $-\operatorname{sign}\left[J_{2}\right]$, which is responsible for the direction of circulating current, in the experssion of circulating current for the first time introduced in J. Yi, J. H. Wei, J. Hong and S.-I. Lee, Phys. Rev. B 65, 033305 (2001).

[14] W. Park and J. Hong, Phys. Rev. B 69, 035319 (2004).

[15] S. Y. Cho, R. H. McKenzie, K. Kang, and C. K. Kim, J. Phys.: Condens. Matter 15, 1147 (2003).

[16] S. Y. Cho and R. H. McKenzie, Phys. Rev. B 71, 045317 (2005).

[17] H. -C. Wu, Y. Guo, X. -Y. Chen and B.-L. Gu, Phys. Rev. B 68, 125330 (2003).

[18] Y.-T. Zhang, Y. Guo and Y.-C. Li, Phys. Rev. B 72, 125334 (2005).

[19] T. Choi, C.-M. Ryu and A. M. Jayannavar, Int. J. Mod. Phys. B 12, 2091 (1998).

[20] R. Citro and F. Romeo, Phys. Rev. B 75, 073306 (2007).

[21] J.-B. Xia, Phys. Rev. B 45, 3593 (1992).

[22] S. Griffith, Trans. Faraday Soc. 49, 650 (1953). 